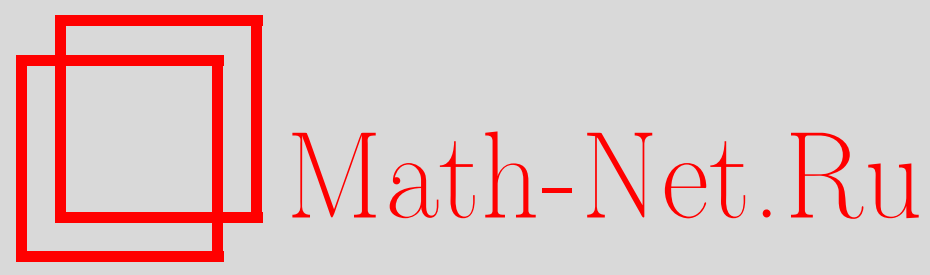

Е. В. Бедулев, О линейной независимости чисел над числовыми полями, Матем. заметки, 1998, том 64, выпуск 4, 506-517

DOI: https://doi.org/10.4213/mzm1425

Использование Общероссийского математического портала Math-Net.Ru подразумевает, что вы прочитали и согласны с пользовательским соглашением http://www.mathnet.ru/rus/agreement

Параметры загрузки:

IP : 3.80 .181 .102

26 апреля 2023 г., 12:15:32

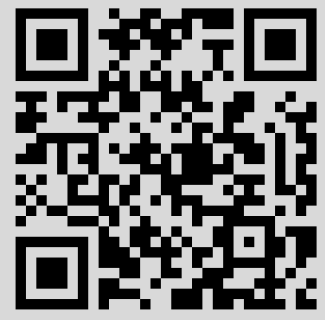




\section{О ЛИНЕЙНОЙ НЕЗАВИСИМОСТИ ЧИСЕЛ НАД ЧИСЛОВЫМИ ПОЛЯМИ}

Е.В. Бедулев

В данной работе рассматривается задача об оценке снизу меры линейной независимости чисел $\theta_{1}, \ldots, \theta_{n}$ при условии, что для последовательности полиномов с целыми алгебраическими коэффициентами известны оценки снизу и сверху в точке $\left(\theta_{1}, \ldots, \theta_{n}\right)$. Используемый метод является обобщением метода Нестеренко на случай произвольного поля алгебраических чисел.

Библиограффия: 9 названий.

В теории трансцендентных чисел известно несколько попыток многомерного обобщения леммы “о плотной последовательности" Гельфонда. Эти обобщения выглядят следующим образом. Пусть $\mathbb{K}$ - числовое поле, $\left(\theta_{1}, \theta_{2}, \ldots, \theta_{n}\right)$ - некоторьй набор чисел, и задано семейство многочленов от $n$ переменных с коэффициентами из кольца $\mathbb{Z}_{\mathbb{K}}$ целых поля $\mathbb{K}$ с контролируемыми степенями и высотами, для значений которых в точке $\theta=\left(\theta_{1}, \ldots, \theta_{n}\right)$ имеются оценки сверху и снизу. Тогда можно оценить снизу степень трансцендентности поля $\mathbb{K}\left(\theta_{1}, \ldots, \theta_{n}\right)$ над $\mathbb{K}$. Впервые оценку подобного вида получил Г.В. Чудновский в работе [1] с помощью последовательного исключения переменных и сведения к одномерному случаю (его доказательство было неполным; впоследствии полные доказательства были опубликованы в [2] и [3]). В 1983 году Ю. В. Нестеренко [4] предложил другой подход, развивая который П. Филлипон [5] получил наилучшую оценку в данной задаче. Тогда же Нестеренко предложил вариант своего метода для случая проблемы линейной независимости чисел над $\mathbb{K}=\mathbb{Q}$ (см. [6]). Настоящая работа посвящена оценкам этого типа в случае произвольного $\mathbb{K}$.

Введем необходимые обозначения. Пусть $\mathbb{K} \subset \mathbb{C}$ - числовое поле и $\mathscr{K}$ - его пополнение, соответствующее этому вложению, т.е. $\mathscr{K}$ есть $\mathbb{C}$ или $\mathbb{R}$. Положим $\delta=[\mathscr{K}: \mathbb{R}]$, $d=[\mathbb{K}: \mathbb{Q}]=r_{1}+2 r_{2}$, где $r_{1}$ - число вещественных, $r_{2}$ - число комплексных вложений поля $\mathbb{K}$.

Для линейной формы $L(x)=\sum_{i=1}^{n} a_{i} x_{i}, a_{i} \in \mathbb{Z}_{\mathbb{K}}$, положим $H(L)=\max _{i} H\left(a_{i}\right)$, где $H\left(a_{i}\right)$ - обычная высота Вейля алгебраического числа $a_{i}$ (подробнее см. п. 2).

Зафиксируем векторное пространство $E$ над $\mathbb{Q}$ размерности $n$ с выделенньм базисом $\left\{e_{1}, \ldots, e_{n}\right\}$. Формулировка (и доказательство) нашей основной теоремы опирается на понятие высоты $H_{c}(N) \mathbb{K}$-рационального подпространства $N$ в $E \otimes \mathscr{K}$. В полной обшности оно вводится в п. 2 , причем для $\mathbb{K}=\mathbb{Q}$ совпадает с определением, данным Нестеренко: если $N$ - некоторое $\mathbb{Q}$-рациональное подпространство в $E \otimes \mathbb{R}$, то $N \cap$

Работа выполнена при частичной поддержке Международного научного фонда (фонда Сороса), грант № 507_s. 
$\left\{\mathbb{Z} e_{1}+\cdots+\mathbb{Z} e_{n}\right\}-$ полная решетка в нем. В качестве $H_{c}(N)$ можно взять объем фундаментального параллелепипеда этой решетки (предполагается, что в $E \otimes \mathbb{R}$ введено скалярное произведение, относительно которого $\left\{e_{1}, \ldots, e_{n}\right\}$ - ортонормированньй базис).

Сформулируем основную теорему.

ОсновНАЯ теОРемА. Пусть вектор $\theta \in E \otimes \mathscr{K}$ фиксирован, $\sigma: \mathbb{N} \rightarrow \mathbb{R}$ - некоторая функиия, удовлетворяющая условиям

$$
\sigma(t+1) \geqslant \sigma(t) \quad \text { npu } t \geqslant t_{0}, \quad \lim _{t \rightarrow+\infty} \sigma(t)=+\infty, \quad \varlimsup_{t \rightarrow+\infty} \frac{\sigma(t+1)}{\sigma(t)}=1,
$$

и имеется набор линейных форм $L_{\nu}(x)=\sum_{i=1} a_{i}^{(\nu)} x_{i}, a_{i}^{(\nu)} \in \mathbb{Z}_{\mathbb{K}}$, такой, что

$$
H\left(L_{\nu}\right) \leqslant e^{\sigma(\nu)}, \quad e^{-\sigma_{1}(\nu)} \leqslant\left|L_{\nu}(\theta)\right| \leqslant e^{-\sigma_{2}(\nu)},
$$

$2 \partial e$

$$
\lim _{t \rightarrow+\infty} \frac{\sigma_{1}(t)}{\sigma(t)}=\tau_{1}, \quad \lim _{t \rightarrow+\infty} \frac{\sigma_{2}(t)}{\sigma(t)}=\tau_{2}, \quad \tau_{1} \geqslant \tau_{2}
$$

Для произвольного $\varepsilon, 0<\varepsilon<\left(\tau_{1}+\delta^{-1}+1\right) / 6$, построим последовательность $\left\{\alpha_{j}\right\}$ из соотношений

$$
\alpha_{0}=1, \quad \frac{\delta^{-1}+\tau_{2}}{\alpha_{j-1}}-\frac{\delta^{-1}+\tau_{1}}{\alpha_{j}}=r_{1}+r_{2}+\varepsilon, \quad j \geqslant 1
$$

Пусть иелое $s \geqslant 0$ таково, что все $\alpha_{j}, j=0, \ldots, s$, определень $и \alpha_{j} \geqslant 1$ для $j=0, \ldots, s$. Тогда существует $H_{0}=H_{0}(\theta, \varepsilon, s, \ldots)$ такое, что для любого $H \geqslant H_{0}$ u любого $\mathbb{K}$-рачионального подпространства $N \subset E \otimes \mathscr{K}$ размерности $s$ над $\mathscr{K}$ неравенства

$$
\rho(\theta, N)<H^{-\alpha_{s}}, \quad H_{c}(N) \leqslant H
$$

несовместны.

ЗАмЕЧАнИЕ. Последовательность $\left\{\alpha_{j}\right\}$ и условие на $s$ в формулировке основной теоремы можно описать явно. А именно,

1) если $\tau_{1}=\tau_{2}$, то

$$
\alpha_{j}=\left(1-j \frac{r_{1}+r_{2}+\varepsilon}{\tau_{1}+\delta^{-1}}\right)^{-1}, j \geqslant 1, \quad s<\frac{\tau_{1}+\delta^{-1}}{r_{1}+r_{2}+\varepsilon}
$$

$2)$ если $\tau_{1}>\tau_{2}$, то, полагая $l=\left(r_{1}+r_{2}+\varepsilon\right) /\left(\tau_{1}-\tau_{2}\right)$, имеем

$$
\alpha_{j}=\left((1+l)\left(\frac{\delta \tau_{2}+1}{\delta \tau_{1}+1}\right)^{j}-l\right)^{-1}, j \geqslant 1, \quad s \ln \frac{\delta \tau_{1}+1}{\delta \tau_{2}+1}<\ln \left(1+\frac{1}{l}\right) .
$$


СлЕДСТВИЕ. Если выполнены условия теоремы, то среди чисел $\theta_{1}, \ldots, \theta_{n}$ имеется по крайней мере $\left(\tau_{1}+\delta^{-1}\right) /\left(r_{1}+r_{2}\right)$ линейно независимых над $\mathbb{K}$ в случае $\tau_{1}=\tau_{2}$ ине менее

$$
\ln \left(1+\frac{\tau_{1}-\tau_{2}}{r_{1}+r_{2}}\right) / \ln \frac{\tau_{1}+\delta^{-1}}{\tau_{2}+\delta^{-1}}
$$

в случае $\tau_{1}>\tau_{2}$.

ДокАЗАТЕЛЬСтво. Предположим, что $\tau_{1}=\tau_{2}$ (случай $\tau_{1}>\tau_{2}$ доказьвается аналогично). Если утверждение следствия неверно, то существует $\mathbb{K}$-рациональное подпространство $N \subset E \otimes \mathscr{K}$ размерности $s$ такое, что $\theta \in N$ и $s<\left(\tau_{1}+\delta^{-1}\right) /\left(r_{1}+r_{2}\right)$. Выберем $\varepsilon>0$ так, чтобы $s<\left(\tau_{1}+\delta^{-1}\right) /\left(r_{1}+r_{2}+\varepsilon\right)$. Применяя к данной ситуации основную теорему, находим, что для любого $H \geqslant H_{0}$ неравенства (3) несовместны. Но $\rho(\theta, N)=0$, что при достаточно больших $H$ противоречиво.

ЗАмЕчАниЕ. Сравним в случае $\mathbb{K}=\mathbb{Q}$ нашу теорему с аналогичной теоремой Нестеренко из [6]. Легко видеть, что при $\tau_{1}=\tau_{2}$ наша оценка совпадает с оценкой Нестеренко, а при $\tau_{1}>\tau_{2}-$ сильнее последней.

Доказательство основной теоремы содержится в п. 3 , п. 1 и п. 2 посвящены доказательству необходимых вспомогательных фактов.

На протяжении всей работы мы пользуемся следующими обозначениями и соглашениями: основное поле $\mathscr{K}$ есть $\mathbb{C}$ или $\mathbb{R}$; если не оговорено обратное, все пространства и подпространства, используемые в работе, рассматриваются над $\mathscr{K} ; \delta=[\mathscr{K}: \mathbb{R}]$ (т.е. $\delta=1$ или $\delta=2) ;\left\langle a_{1}, \ldots, a_{k}\right\rangle$ - подпространство, натянутое на векторы $a_{1}, \ldots, a_{k}$. Под скалярньм произведением в некотором $\mathscr{K}$-векторном пространстве мы подразумеваем евклидово скалярное произведение в случае $\mathscr{K}=\mathbb{R}$ и эрмитово скалярное произведение в случае $\mathscr{K}=\mathbb{C}$. Предполагая, что $\mathscr{K}$-векторное пространство $V$ снабжено таким скалярньм произведением, обозначим через $M^{\perp}$ ортогональное дополнение к подпространству $M \subset V$, через $\operatorname{pr}_{M} \theta$ ортогональную проекцию $\theta \in V$ на подпространство $M$;

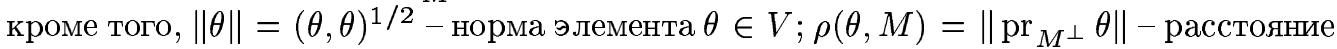
от точки $\theta \in V$ до подпространства $M$. В случае $\mathscr{K}=\mathbb{R}$ обозначим через $\varphi(a, b)$ угол между векторами $a, b \in V, 0 \leqslant \varphi \leqslant \pi$, а через $\varphi(l, m)$ угол между прямыми $l, m \subset V$, $0 \leqslant \varphi \leqslant \pi / 2$. Более общо, $\varphi(l, M)=\varphi\left(l, \operatorname{pr}_{M} l\right)-$ угол между прямой $l \subset V$ и подпространством $M \subset V$.

1. Линейная алгебра. Пусть $V$ - векторное пространство над полем $\mathscr{K}$ размерности $n$, снабженное скалярным произведением. Для нас существенно то, что это скалярное произведение на $E$ индуцирует скалярные произведения на внешних степенях $\bigwedge^{k} V, 1 \leqslant k \leqslant n$. А именно, скалярное произведение на $V$ определяет отождествление $V \cong \overline{V^{*}}$ с пространством антилинейных функционалов на $V$ (черта сверху означает комплексное сопряжение и существенна только в случае $\mathscr{K}=\mathbb{C}$ ), а значит, и отождествление $\bigwedge^{k} V \cong \bigwedge^{k} \overline{V^{*}} \cong \overline{\bigwedge^{k} V^{*}}$, т.е. некоторую билинейную (полуторалинейную в случае $\mathscr{K}=\mathbb{C}$ ) невырожденную форму на $\bigwedge^{k} V$. Отсюда следует координатное описание соответствующего скалярного произведения. Пусть $\left\{a_{1}, \ldots, a_{k}\right\},\left\{b_{1}, \ldots, b_{k}\right\}$ - два набора векторов из $V$,

$$
\left(a_{1}, \ldots, a_{k}\right)=A^{t}\left(e_{1}, \ldots, e_{n}\right), \quad\left(b_{1}, \ldots, b_{k}\right)=B^{t}\left(e_{1}, \ldots, e_{n}\right)
$$


- их разложения по ортонормированному базису $\left\{e_{1}, \ldots, e_{k}\right\}, A, B-(n \times k)$-матрицы с коэффициентами из $\mathscr{K}$. Тогда

$$
\left(a_{1} \wedge \cdots \wedge a_{k}, b_{1} \wedge \cdots \wedge b_{k}\right)=\sum_{I} A_{I} \overline{B_{I}}
$$

где $I$ пробегает все наборы из $k$ столбцов в $(n \times k)$-матрице, $A_{I}, B_{I}$ - соответствующие миноры. Заметим, что отсюда следует положительная определенность нашей билинейной формы, т.е. она является скалярным произведением на $\bigwedge^{k} V$.

Заметим, что если обозначить через $\operatorname{Vol}\left(a_{1}, \ldots, a_{k}\right)$ объем параллелепипеда, натянутого на векторы $a_{1}, \ldots, a_{k} \in V$, то

$$
\operatorname{Vol}\left(a_{1}, \ldots, a_{k}\right)=\left\|a_{1} \wedge \cdots \wedge a_{k}\right\|_{\wedge^{k} V}=\left(a_{1} \wedge \cdots \wedge a_{k}, a_{1} \wedge \cdots \wedge a_{k}\right)^{1 / 2}
$$

(можно считать это определением объема).

Отметим несколько хорошо известных свойств объема $\operatorname{Vol}\left(a_{1}, \ldots, a_{k}\right)$, которые понадобятся нам в дальнейшем.

ПРЕДЛОЖЕНИЕ 1. Справедливы следующие утверждения:

1) $\operatorname{Vol}\left(a_{1}, \ldots, a_{k}\right)=\left(\operatorname{det}\left|\left(a_{i}, a_{j}\right)\right|_{i, j=1, \ldots, k}\right)^{1 / 2}$;

2) $\operatorname{Vol}\left(a_{1}, \ldots, a_{k}\right)=0$ тогда и только тогда, когда векторы $a_{1}, \ldots, a_{k}$ линейно зависимы;

3) $\operatorname{Vol}\left(a_{1}, \ldots, a_{k}\right)=\rho\left(a_{k}, M\right) \operatorname{Vol}\left(a_{1}, \ldots, a_{k-1}\right)$, әде $M=\left\langle a_{1}, \ldots, a_{k-1}\right\rangle$;

4) $\operatorname{Vol}\left(a_{1}, \ldots, a_{r}, b_{1}, \ldots, b_{s}\right) \leqslant \operatorname{Vol}\left(a_{1}, \ldots, a_{r}\right) \operatorname{Vol}\left(b_{1}, \ldots, b_{s}\right)$, при әтом если наборы $\left\{a_{1}, \ldots, a_{r}\right\} u\left\{b_{1}, \ldots, b_{s}\right\}$ состоят из линейно независимых векторов, то равенство имеет место тогда и только тогда, когда подпространства $\left\langle a_{1}, \ldots, a_{r}\right\rangle u\left\langle b_{1}, \ldots, b_{s}\right\rangle$ ортогональны;

5) $\operatorname{Vol}\left(a_{1}, \ldots, a_{k}\right) \leqslant\left\|a_{1}\right\| \cdots\left\|a_{k}\right\|$.

ДокАЗАТЕЛЬСтво можно найти, например, в [7]. Утверждение 5) следует из утверждения 4).

Пусть $M, N$ - два подпространства в $V$. Мы хотим определить величину $\eta(M, N)$, $0 \leqslant \eta(M, N) \leqslant 1$, измеряющую "угол" между подпространствами $M$ и $N$. Рассмотрим сначала случай $M \cap N=0$. Пусть $\left\{a_{1}, \ldots, a_{r}\right\},\left\{b_{1}, \ldots, b_{s}\right\}$ - произвольные базисы в $M, N$ соответственно. Положим

$$
\eta(M, N)=\frac{\operatorname{Vol}\left(a_{1}, \ldots, a_{r}, b_{1}, \ldots, b_{s}\right)}{\operatorname{Vol}\left(a_{1}, \ldots, a_{r}\right) \operatorname{Vol}\left(b_{1}, \ldots, b_{s}\right)} .
$$

Из предложения 1 следует, что $0 \leqslant \eta(M, N) \leqslant 1$, причем $\eta(M, N) \neq 0$ в предположении $M \cap N=0$ и $\eta(M, N)=1$, если и только если $M$ ортогонально $N$. Остается проверить, что величина $\eta(M, N)$ не зависит от выбора базисов в $M$ и $N$. Проще всего убедиться в этом следующим образом: согласно определению

$$
\eta(M, N)=\frac{\left\|a_{1} \wedge \cdots \wedge a_{r} \wedge b_{1} \wedge \cdots \wedge b_{s}\right\|}{\left\|a_{1} \wedge \cdots \wedge a_{r}\right\| \cdot\left\|b_{1} \wedge \cdots \wedge b_{s}\right\|}
$$

и, как хорошо известно, элементы $a_{1} \wedge \cdots \wedge a_{r} \in \wedge^{r} V$ и $b_{1} \wedge \cdots \wedge b_{s} \in \bigwedge^{s} V$ восстанавливаются по подпространствам $M$ и $N$ однозначно с точностью до пропорциональности. 
Пусть теперь $M \cap N=T$ - необязательно нулевое подпространство, но $M \not \subset N$ и $N \not \subset M$. Тогда подпространства $M_{1}=M \cap T^{\perp}$ и $N_{1}=N \cap T^{\perp}$ имеют нулевое пересечение, и можно рассматривать величину

$$
\eta(M, N)=\eta\left(M_{1}, N_{1}\right)
$$

в смысле предыдущего определения или, что то же самое,

$$
\eta(M, N)=\frac{\operatorname{Vol}\left(a_{1}, \ldots, a_{r}, b_{1}, \ldots, b_{s}, c_{1}, \ldots, c_{t}\right) \operatorname{Vol}\left(c_{1}, \ldots, c_{t}\right)}{\operatorname{Vol}\left(a_{1}, \ldots, a_{r}, c_{1}, \ldots, c_{t}\right) \operatorname{Vol}\left(b_{1}, \ldots, b_{s}, c_{1}, \ldots, c_{t}\right)}
$$

где $\left\{c_{1}, \ldots, c_{t}\right\},\left\{a_{1}, \ldots, a_{r}\right\},\left\{b_{1}, \ldots, b_{s}\right\}$ - произвольные базисы в $T, M_{1}$ и $N_{1}$ соответственно.

Рассмотрим теперь примеры, поясняющие определение величины $\eta(M, N)$. Пусть $\mathscr{K}=\mathbb{R}$ и $l, m$ - две прямые в $V$. Тогда, очевидно, $\eta(l, m)=\sin \varphi(l, m)$ в силу хорошо известной формулы $\operatorname{Vol}(a, b)= \pm\|a\| \cdot\|b\| \sin \varphi(a, b)$ для произвольных векторов $a, b \in V$.

Более общо, пусть $M$ - подпространство с базисом $a_{1}, \ldots, a_{r}, l$ - прямая с образующим вектором $\theta$. Тогда

$$
\eta(l, M)=\frac{\operatorname{Vol}\left(a_{1}, \ldots, a_{r}, \theta\right)}{\operatorname{Vol}\left(a_{1}, \ldots, a_{r}\right)\|\theta\|}=\frac{\rho(\theta, M)}{\|\theta\|}=\sin \varphi(l, M)
$$

в силу предложения 1 . Таким образом, величина $\eta(M, N)$ действительно измеряет угол между $M$ и $N$.

Случай $M \cap N \neq 0$ иллюстрируется следуюшим примером. Пусть $M$ и $N$ - две плоскости в трехмерном пространстве. Тогда определение величины $\eta(M, N)$, очевидно, согласовано с обычным понятием двугранного угла между ними.

В случае $\mathscr{K}=\mathbb{C}$ наряду с комплексным пространством $V$ размерности $n$, снабженным эрмитовым скалярным произведением $(\cdot, \cdot)$, можно рассматривать его овеществление $V_{\mathbb{R}}$ размерности $2 n$, снабженное евклидовьм скалярным произведением $(\cdot, \cdot)_{\mathbb{R}}=$ $\operatorname{Re}(\cdot, \cdot)$. В частности, если $M, N$ - комплексные подпространства, то определены "углы" $\eta(M, N)$ (в эрмитовом пространстве $E$ ) и $\eta_{\mathbb{R}}(M, N)$ (в евклидовом пространстве $\left.V_{\mathbb{R}}\right)$. Связь между этими величинами выражается формулой $\eta_{\mathbb{R}}(M, N)=\eta(M, N)^{2}$, которая вытекает из следующей известной леммы.

Лемма 1. Справедливо тождество

$$
\operatorname{Vol}\left(a_{1}, \ldots, a_{k}\right)^{2}=\operatorname{Vol}_{\mathbb{R}}\left(a_{1}, \ldots, a_{k}, i a_{1}, \ldots, i a_{k}\right)
$$

Здесь в левой части стоит обгем в әрмитовом пространстве $V$, а в правойобгем в евклидовом пространстве $V_{\mathbb{R}}, i$ - мнимая единица.

Докажем две вспомогательные леммы.

Лемма 2. Пусть $M_{1}, M, N_{1}, N$ - подпространства в $V, M \cap N=0$, причем $M_{1} \subset M, N_{1} \subset N$. Тогда $\eta\left(M_{1}, N_{1}\right) \geqslant \eta(M, N)$. 
ДокАЗАТЕЛЬСтво. Выберем в $M$ и $N$ ортонормированные базисы $\left\{a_{1}, \ldots, a_{r}\right\}$ и $\left\{b_{1}, \ldots, b_{s}\right\}$ так, что $\left\{a_{1}, \ldots, a_{k}\right\}$ и $\left\{b_{1}, \ldots, b_{l}\right\}$ - базисы в $M_{1}, N_{1}$ соответственно, $k \leqslant r$, $l \leqslant s$. Тогда

$$
\begin{aligned}
\eta(M, N) & =\frac{\operatorname{Vol}\left(a_{1}, \ldots, a_{r}, b_{1}, \ldots, b_{s}\right)}{\operatorname{Vol}\left(a_{1}, \ldots, a_{r}\right) \operatorname{Vol}\left(b_{1}, \ldots, b_{s}\right)} \\
& \leqslant \frac{\operatorname{Vol}\left(a_{1}, \ldots, a_{k}, b_{1}, \ldots, b_{l}\right) \cdot \prod_{i=k+1}^{r}\left\|a_{i}\right\| \cdot \prod_{j=l+1}^{s}\left\|b_{j}\right\|}{1 \cdot 1} \\
& =\frac{\operatorname{Vol}\left(a_{1}, \ldots, a_{k}, b_{1}, \ldots, b_{l}\right)}{\operatorname{Vol}\left(a_{1}, \ldots, a_{k}\right) \operatorname{Vol}\left(b_{1}, \ldots, b_{l}\right)}=\eta\left(M_{1}, N_{1}\right),
\end{aligned}
$$

что и требовалось доказать.

Лемма 3. Пусть $\mathscr{K}=\mathbb{R} u l_{1}, l_{2}, l_{3}-$ три прямые в $V$. Тогда

$$
\sin \varphi\left(l_{1}, l_{3}\right) \leqslant \sin \varphi\left(l_{1}, l_{2}\right)+\sin \varphi\left(l_{2}, l_{3}\right) .
$$

ДОКАЗАТЕЛЬСТвО. Прежде всего, если $l_{1}, l_{2}, l_{3}$ лежат в одной плоскости, то

$$
\varphi\left(l_{1}, l_{3}\right)= \pm \varphi\left(l_{1}, l_{2}\right) \pm \varphi\left(l_{2}, l_{3}\right)
$$

при некотором выборе знаков “ \pm ", и требуемое неравенство следует из формулы синуса суммы. Рассмотрим теперь общий случай. Пусть $M=l_{1}+l_{2}$ и $l_{3}^{\prime}=\operatorname{pr}_{M} l_{3}$. Если мы проверим, что для любой прямой $l \subset M$

$$
\varphi\left(l, l_{3}^{\prime}\right) \leqslant \varphi\left(l, l_{3}\right)
$$

то по уже доказанному

$$
\sin \varphi\left(l_{1}, l_{2}\right) \leqslant \sin \varphi\left(l_{1}, l_{3}^{\prime}\right)+\sin \varphi\left(l_{3}^{\prime}, l_{2}\right) \leqslant \sin \varphi\left(l_{1}, l_{3}\right)+\sin \varphi\left(l_{3}, l_{2}\right),
$$

следовательно, получим требуемое.

Итак, осталось проверить неравенство (5). Пусть $e_{3}, e$ - орты прямых $l_{3}$ и $l$ соответственно такие, что $\left(e_{3}, e\right) \geqslant 0$. Пусть $e_{3}^{\prime}=\mathrm{pr}_{M} e_{3}$. Тогда $l_{3}^{\prime}=\mathbb{R} e_{3}^{\prime}$ и

$$
\cos \varphi\left(e_{3}, e\right)=\left(e_{3}, e\right)=\left(e_{3}^{\prime}, e\right)=\cos \varphi\left(e_{3}^{\prime}, e\right) \cdot\left\|e_{3}^{\prime}\right\| \leqslant \cos \varphi\left(e_{3}^{\prime}, e\right)
$$

так как $\left\|e_{3}^{\prime}\right\| \leqslant\left\|e_{3}\right\|=1$. Лемма доказана.

ПРЕДЛОЖЕНИЕ 2. Пусть $M, N-$ подпространства в $V, \theta \in V$. Тогда

$$
\eta(M, N)^{\delta} \rho(\theta, M \cap N) \leqslant \rho(\theta, M)+\rho(\theta, N),
$$

где $\delta=[\mathscr{K}: \mathbb{R}]$. 
ДокаЗАТЕЛьСтво. Так как $\rho(\theta, M)=\rho_{\mathbb{R}}\left(\theta, M_{\mathbb{R}}\right)$ и $\eta(M, N)^{2}=\eta_{\mathbb{R}}(M, N)$ в случае $\mathscr{K}=\mathbb{C}$, достаточно рассмотреть случай $\mathscr{K}=\mathbb{R}$. Пусть $T=M \cap N \neq 0$ и $\theta_{1}=\operatorname{pr}_{T^{\perp}} \theta$. Тогда $\theta-\theta_{1} \in T=M \cap N$, откуда $\operatorname{pr}_{M^{\perp}} \theta=\operatorname{pr}_{M^{\perp}} \theta_{1}$ и $\operatorname{pr}_{N^{\perp}} \theta=\operatorname{pr}_{N^{\perp}} \theta_{1}$. Теперь искомое неравенство преобразуется к виду

$$
\eta\left(M_{1}, N_{1}\right)\left\|\theta_{1}\right\| \leqslant \rho\left(\theta_{1}, M_{1}\right)+\rho\left(\theta_{1}, N_{1}\right),
$$

где $M_{1}=M \cap T^{\perp}, N_{1}=N \cap T^{\perp}$. Поэтому достаточно рассмотреть случай $M \cap N=0$. Согласно (4) наше неравенство эквивалентно неравенству $\eta(M, N) \leqslant \eta(l, M)+\eta(l, N)$, где $l=\mathbb{R} \theta$. Если обозначить $l_{M}=\operatorname{pr}_{M} l, l_{N}=\operatorname{pr}_{N} l$, то в силу лемм 2 и 3 имеем

$$
\eta(M, N) \leqslant \eta\left(l_{M}, l_{N}\right) \leqslant \eta\left(l_{M}, l\right)+\eta\left(l_{N}, l\right)=\eta(l, M)+\eta(l, N),
$$

что и требовалось доказать.

2. Высоты. В дальнейшем мы будем придерживаться следующих обозначений.

Пусть $\mathbb{K}$ - фиксированное числовое поле абсолютной степени $d=[\mathbb{K}: \mathbb{Q}]$. Будем рассматривать различные вложения $\mathbb{K} \subset \mathbb{C}$. Зафиксируем в каждой паре комплексно-сопряженных вложений какое-нибудь одно. Обозначим элементы получившейся системы вложений через $v$ и назовем их архимедовыми нормированиями поля $\mathbb{K}$. Пусть $x^{v}$ - образ элемента $x \in \mathbb{K}$ при вложении $v,|x|_{v}=\left|x^{v}\right|$ (обычный комплексньй модуль). Пусть, как обычно, $r_{1}$ - число вещественных вложений $v$ и $r_{2}-$ число комплексных, $d=r_{1}+2 r_{2}$.

Пусть $\mathbb{K}_{v}-$ пополнение $\mathbb{K}$ по нормированию $v, \delta_{v}=\left[\mathbb{K}_{v}: \mathbb{R}\right]$.

Выделим некоторое вложение $\mathbb{K} \subset \mathbb{C}$, соответствующее архимедово значение обозначим через $w$ и отождествим поле $\mathscr{K}$ (см. п. 1 ) с $\mathbb{K}_{w}$. Таким образом, $\delta=\delta_{w}$.

Пусть теперь $E$ - векторное пространство над $\mathbb{Q}$ с выделенньм базисом $\left\{e_{1}, \ldots, e_{n}\right\}$, $\mathbb{P} E$ - соответствующее проективное пространство, $\alpha=\left(\alpha_{1}: \cdots: \alpha_{n}\right) \in \mathbb{P} E(\mathbb{K}), \alpha_{i} \in \mathbb{K}$. Напомним (см. $[8$, гл. 3$])$, что высота Вейля определяется соотношением

$$
H(\alpha)=\frac{1}{\operatorname{Norm}\left(\alpha_{1}, \ldots, \alpha_{n}\right)} \prod_{v} \max _{i}\left|\alpha_{i}\right|^{\delta_{v}} .
$$

Здесь $\left(\alpha_{0}, \ldots, \alpha_{n}\right)$ - дробный идеал в $\mathbb{K}$, порожденный $\alpha_{0}, \ldots, \alpha_{n}$, a Norm - обычная абсолютная норма дробного идеала.

Однако, нам понадобится другая высота, а именно:

$$
H_{c}(\alpha)=\frac{1}{\operatorname{Norm}\left(\alpha_{1}, \ldots, \alpha_{n}\right)} \prod_{v}\left(\left|\alpha_{0}\right|_{v}^{2}+\cdots+\left|\alpha_{n}\right|_{v}^{2}\right)^{\delta_{v} / 2} .
$$

Очевидно, что это определение не зависит от выбора однородных координат $\alpha \in \mathbb{P} E(\mathbb{K})$. Заметим также, что $H_{c}(\alpha) \geqslant H(\alpha) \geqslant 1$ (см. [8, гл. 3]).

Будем говорить, что $\mathscr{K}$-векторное подпространство $M \subset E \otimes \mathscr{K}$ определено над $\mathbb{K}$ (или $\mathbb{K}$-рационально), если его можно задать набором линейных форм с коэффициентами из $\mathbb{K}$ или, что то же самое, если существует базис $\left\{a_{1}, \ldots, a_{k}\right\}$ в $M$ такой, что для разложения

$$
\left(a_{1}, \ldots, a_{k}\right)=A^{t}\left(e_{1}, \ldots, e_{n}\right)
$$


по базису $\left\{e_{1}, \ldots, e_{n}\right\}$ вьполнено $A \in \operatorname{Mat}(n \times k, \mathbb{K})$.

Заметим, что $\mathbb{K}$-рациональное подпространство $M$ определяет подпространства $M_{v}$ во всех $E \otimes \mathbb{K}_{v}$ (для этого вложения $v: \mathbb{K} \subset \mathbb{C}$ применяются к матрице $A$ ).

Введем в $E \otimes \mathscr{K}$ скалярное произведение так, чтобы базис $\left\{e_{1}, \ldots, e_{n}\right\}$ был ортонормированным. Тогда скалярные произведения с тем же свойством будут определены во всех $E \otimes \mathbb{K}_{v}$, т.е. будут определены объемы и углы (см. п. 1). Если $\left\{a_{1}, \ldots, a_{k}\right\}-$ базис в $M$, удовлетворяющий условию (6), то будем писать $\operatorname{Vol}_{v}\left(a_{1}, \ldots, a_{k}\right)$ вместо $\operatorname{Vol}\left(a_{1}^{v}, \ldots, a_{k}^{v}\right)$ и $\eta_{v}(M, N)$ вместо $\eta\left(M_{v}, N_{v}\right)$, где $M, N-\mathbb{K}$-рациональные подпространства в $E \otimes \mathscr{K}$.

ЗАмечАниЕ. Легко видеть, что если $M \perp N$, то $M^{v} \perp N^{v}$ для всех $v$.

Пусть $M$ - векторное подпространство размерности $k$ в $E \otimes \mathscr{K}$, определенное над $\mathbb{K}$.

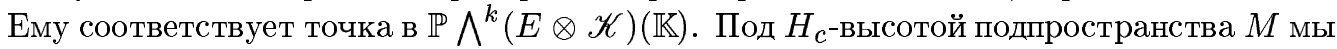
понимаем $H_{c}$-высоту соответствующей точки (при этом предполагается, что в $k$-й внешней степени пространства $E \otimes \mathscr{K}$ выбран базис $\left.\left\{e_{i_{1}} \wedge \cdots \wedge e_{i_{k}}\right\}_{1 \leqslant i_{1}}<\cdots<i_{k} \leqslant n\right)$. Координатное описание соответствующей высоты таково: если $\left\{a_{1}, \ldots, a_{k}\right\}$ - базис $M$, удовлетворяющий условию (6), то

$$
H_{c}(M)=\frac{1}{\operatorname{Norm}\left(\mathscr{M}\left(a_{1}, \ldots, a_{k}\right)\right)} \prod_{v} \operatorname{Vol}_{v}\left(a_{1}, \ldots, a_{k}\right)^{\delta_{v}} .
$$

Здесь $\mathscr{M}\left(a_{1}, \ldots, a_{k}\right)$ - дробный идеал в $\mathbb{K}$, порожденньй $(k \times k)$-минорами матрицы $A$ из (6).

В дальнейшем нам понадобится элементарная лемма, являющаяся своеобразным обобщением разложения Лапласа для определителей.

Пусть $A$ - квадратная $(n \times n)$-матрица; $I \cup I^{\prime} \cup I^{\prime \prime}$ - некоторое разбиение множества столбцов матрицы $A$ на наборы $I, I^{\prime}, I^{\prime \prime}$ и $J \cup J^{\prime} \cup J^{\prime \prime}$ - некоторое разбиение множества строк матрицы $A$ на наборы $J, J^{\prime}, J^{\prime \prime}$. О столбцах, лежащих в наборе $I$, будем говорить как о $I$-столбцах, аналогично определяются $J$-строки; $A_{J}^{I}$ - подматрицы матрицы $A$, образованные $I$-столбцами и $J$-строками в естественном порядке следования. Обозначим через $\operatorname{deg} I$ число элементов в наборе $I$, через $\left|I^{\prime}\right|$ и $\left|J^{\prime}\right|$ сумму всех номеров столбцов (строк) в соответствующем наборе в матрище $A_{J^{\prime} \cup J^{\prime \prime}}^{I^{\prime \prime}}$.

Лемма 4. Пусть наборь $I, J, J^{\prime} u J^{\prime \prime}$ фиксированьь, $\operatorname{deg} I=\operatorname{deg} J$. Тогда справедливо тождество

$$
\operatorname{det} A_{J}^{I} \cdot \operatorname{det} A=\sum_{I^{\prime}, I^{\prime \prime}}(-1)^{\left|I^{\prime}\right|+\left|J^{\prime}\right|} \operatorname{det} A_{J \cup J^{\prime}}^{I \cup \operatorname{det}} A_{J \cup J^{\prime \prime}}^{I \cup I^{\prime \prime}},
$$

где суммирование ведется по наборам столбцов $I^{\prime}$ и $I^{\prime \prime}$ матрицы $A$ таким, что $I \cup I^{\prime} \cup I^{\prime \prime}-$ разбиение мнохества всех столбиов $u \operatorname{deg} I^{\prime}=\operatorname{deg} J^{\prime}, \operatorname{deg} I^{\prime \prime}=\operatorname{deg} J^{\prime \prime}$.

ДокАЗАТЕЛЬСТво. Заметим, что искомая формула остается инвариантной при следующих преобразованиях:

а) вычитание из $I$-столбца кратного другому $I$-столбцу;

$\left.\mathrm{a}^{\prime}\right)$ вычитание из $J$-строки кратной другой $J$-строке;

b) перестановка двух $I$-столбцов; 
$\left.\mathrm{b}^{\prime}\right)$ перестановка двух $J$-строк;

c) вычитание из $J^{\prime}$-строки ( $J^{\prime \prime}$-строки) кратной $J$-строке;

d) выгитание из $J^{\prime}$-строки ( $J^{\prime \prime}$-строки) кратной другой $J^{\prime}$-строке ( $J^{\prime \prime}$-строке);

е) перестановка двух $J^{\prime}$-строк $\left(J^{\prime \prime}\right.$-строк).

Прежде всего, применяя операции $\left.\left.\left.\mathrm{a}), \mathrm{a}^{\prime}\right), \mathrm{b}\right), \mathrm{b}^{\prime}\right)$, мы можем привести матрицу $A_{J}^{I}$ к виду $\operatorname{diag}(1, \ldots, 1,0, \ldots, 0)$. Обозначим набор тех столбцов матрицы $A_{J}^{I}$, в которых стоят единищы, через $I_{0}$. Рассмотрим два случая.

1) Пусть $I_{0} \neq \varnothing$. Тогда, применяя операцию с), мы можем сделать нулевьми столбцы $I_{0}$ в матрице $A_{J^{\prime}}^{I} \cup J^{\prime \prime}$. Благодаря этому все определители в формуле $(7)$ можно разложить по $I_{0}$-столбцам. Если $I_{0}=I$, то полученная формула будет в точности совпадать с разложением Лапласа, что и доказывает ее в этом случае. Если же $I_{0} \neq I$, то мы получаем ту же задачу, но с $I_{0}=\varnothing$.

2) Пусть $I_{0}=\varnothing$. Тогда $A_{J}^{I}=0$, и надо доказать, что правая часть (7) обращается в 0 . Применяя операции d) и е), все строки в матрищах $A_{J^{\prime}}^{I}$ и $A_{J^{\prime \prime}}^{I}$ можем привести к виду $(0, \ldots, 0,1,0, \ldots, 0)$ (с единицей на некотором месте). Если теперь в матрицах $A_{J^{\prime}}^{I}$ и $A_{J^{\prime \prime}}^{I}$ имеются нулевые столбцы, то формула $(7)$ тавтологична (все слагаемые обращаются в 0 ). Поэтому единственным нетривиальньг случаем является случай $\operatorname{deg} J^{\prime}=\operatorname{deg} J^{\prime \prime}=\operatorname{deg} J, A_{J^{\prime}}^{I}=A_{J^{\prime \prime}}^{I}=E$. Разложив все определители в правой части (7) по $I$-столбцам, получим хорошо известную формулу (см., например, [9])

$$
\sum(-1)^{\left|I^{\prime}\right|} \operatorname{det} A_{J}^{I^{\prime}} \cdot \operatorname{det} A_{J}^{I^{\prime \prime}}=0,
$$

где суммирование ведется так же, как и в (7), $\operatorname{deg} I^{\prime}=\operatorname{deg} I^{\prime \prime}=\operatorname{deg} J$.

ПРЕДЛОЖЕНИЕ 3. Пусть $M, N-$ два подпространства в $E \otimes \mathscr{K}$, определенные над $\mathbb{K}$, такие, что $M \not \subset N$ и $N \not \subset M$. Пусть $M+N=E \otimes \mathscr{K}$. Тогда

$$
\eta(M, N)^{\delta}=\eta_{w}(M, N)^{\delta_{w}} \geqslant \frac{H_{c}(M \cap N)}{H_{c}(M) H_{c}(N)} .
$$

ДокАЗАТЕльСтво. Положим $T=M \cap N, M_{1}=M \cap T^{\perp}, N_{1}=N \cap T^{\perp}$.

Выберем базисы $\left\{c_{1}, \ldots, c_{t}\right\}$ в $T,\left\{a_{1}, \ldots, a_{r}\right\}$ в $M_{1},\left\{b_{1}, \ldots, b_{s}\right\}$ в $N_{1}$, определенные над $\mathbb{K}$. По определению имеем

$$
\begin{aligned}
\eta_{w}(M, N)^{\delta_{w}} & \\
& \geqslant \prod_{v} \eta_{v}(M, N)^{\delta_{v}}=\prod_{v}\left(\frac{\operatorname{Vol}_{v}\left(a_{1}, \ldots, a_{r}, b_{1}, \ldots, b_{s}, c_{1}, \ldots, c_{t}\right) \operatorname{Vol}_{v}\left(c_{1}, \ldots, c_{t}\right)}{\operatorname{Vol}_{v}\left(a_{1}, \ldots, a_{r}, c_{1}, \ldots, c_{t}\right) \operatorname{Vol}_{v}\left(b_{1}, \ldots, b_{s}, c_{1}, \ldots, c_{t}\right)}\right)^{\delta_{v}} \\
& =\frac{H_{c}(M+N) H_{c}(M \cap N)}{H_{c}(M) H_{c}(N)} \operatorname{Norm} \frac{\mathscr{M}\left(a_{1}, \ldots, a_{r}, b_{1}, \ldots, b_{s}, c_{1}, \ldots, c_{t}\right) \mathscr{M}\left(c_{1}, \ldots, c_{t}\right)}{\mathscr{M}\left(a_{1}, \ldots, a_{r}, c_{1}, \ldots, c_{t}\right) \mathscr{M}\left(b_{1}, \ldots, b_{s}, c_{1}, \ldots, c_{t}\right)} \\
& \geqslant \frac{H_{c}(M \cap N)}{H_{c}(M) H_{c}(N)},
\end{aligned}
$$

так как под нормой стоит дробньй идеал в $\mathbb{K}$, являющийся, очевидно, цельп. В самом деле, поскольку $M+N=E \otimes \mathscr{K}$, включение

$$
\begin{aligned}
& \mathscr{M}\left(a_{1}, \ldots, a_{r}, b_{1}, \ldots, b_{s}, c_{1}, \ldots, c_{t}\right) \mathscr{M}\left(c_{1}, \ldots, c_{t}\right) \\
& \quad \subset \mathscr{M}\left(a_{1}, \ldots, a_{r}, c_{1}, \ldots, c_{t}\right) \mathscr{M}\left(b_{1}, \ldots, b_{s}, c_{1}, \ldots, c_{t}\right)
\end{aligned}
$$

следует из леммы 4. 
ЗАмечАниЕ. Неравенство (8) также имеет место в случае $M+N \neq E \otimes \mathscr{K}$. Для этого необходимо лишь соответствующее обобщение леммы 4.

СлЕДСТВИЕ. Справедливы неравенства

$$
\begin{gathered}
H_{c}(M \cap N) \leqslant H_{c}(M) H_{c}(N), \\
H_{c}(M \cap N) \rho(\theta, M \cap N) \leqslant H_{c}(M) H_{c}(N)(\rho(\theta, M)+\rho(\theta, N)), \quad \theta \in E \otimes \mathscr{K} .
\end{gathered}
$$

ДокАЗАТЕЛЬСТво. Неравенство (9) очевидно, неравенство (10) следует из предложений 2 и 3.

3. Приложения к диофантовым приближениям. Этот пункт посвящен доказательству основной теоремы. Мы придерживаемся обозначений, введенных в п. 2. В частности, $E$ - векторное пространство над $\mathbb{Q}$ размерности $n$ с выделенным базисом $\left\{e_{1}, \ldots, e_{n}\right\}$.

Лемма 5. Пусть $L(x)=\sum_{i=1}^{n} a_{i} x_{i}$-линейная форма с коэффициентами из $\mathbb{Z}_{\mathbb{K}}$, $M$ - определяемая ею гиперплоскость, $\theta \in E \otimes \mathscr{K}$. Тогда

$$
\begin{gathered}
H_{c}(M)^{\delta^{-1}} \rho(\theta, M) \leqslant n^{d / 2}|L(\theta)| H(L)^{\delta^{-1}\left(r_{1}+r_{2}-1\right)} \\
H_{c}(M) \leqslant n^{d / 2} H(L)^{r_{1}+r_{2}} \\
|L(\theta)| \leqslant n^{1 / 2} H(L)^{\delta^{-1}} \rho(\theta, M) .
\end{gathered}
$$

ДоКАЗАТЕЛЬСТВО. Согласно [8, гл. 3]

$$
\left|a_{i}\right|_{v} \leqslant H\left(a_{i}\right)^{\delta_{v}^{-1}} \leqslant H(L)^{\delta_{v}^{-1}}
$$

для любых $k, v$. Далее,

$$
\begin{gathered}
H_{c}(M) \leqslant \prod_{v}\left(\left|a_{1}\right|_{v}^{2}+\cdots+\left|a_{n}\right|_{v}^{2}\right)^{\delta_{v} / 2}, \\
\rho(\theta, M)=\frac{|L(\theta)|}{\left(\left|a_{1}\right|_{w}^{2}+\cdots+\left|a_{n}\right|_{w}^{2}\right)^{1 / 2}} .
\end{gathered}
$$

Применяя в (15) оценку (14), мы сразу получаем (12). Аналогично, оценив в (16) $\left|a_{i}\right|_{w} \leqslant H(L)^{\delta^{-1}}$, мы получаем (13). Наконец, используя (15) и (16), получаем

$$
H_{c}(M)^{\delta^{-1}} \rho(\theta, M) \leqslant|L(\theta)| \cdot \prod_{v \neq w}\left(\left|a_{1}\right|_{v}^{2}+\cdots+\left|a_{n}\right|_{v}^{2}\right)^{\delta_{v} /(2 \delta)} .
$$

Произведение содержит $r_{1}+r_{2}-1$ множителей, и применение оценки (14) дает неравенство (11).

ДОКАЗАТЕЛЬСТВО ОСНОВНОЙ ТЕОРЕМЫ. Применим индукцию по $s$. При $s=0$ все очевидно: $N=0$ и можно положить $H_{0}=\|\theta\|$. 
Пусть для $s-1$ теорема верна. Докажем ее для $s$, рассуждая от противного. Пусть найдутся $\mathbb{K}$-рациональное подпространство $N \subset E \otimes \mathscr{K}$ размерности $s$ и число $H$ такие, что вьполняются неравенства (3). Покажем, что это приводит к противоречию при больших $H_{0}$.

Пусть $\nu$ - наименьшее натуральное число такое, что

$$
\exp \left(-\left(\tau_{2}+\frac{1}{\delta}-\alpha_{s-1}\left(r_{1}+r_{2}+\frac{\varepsilon}{2}\right)\right) \sigma(\nu)\right) \leqslant H^{-\alpha_{s-1}}
$$

Такое число существует в силу свойств функции $\sigma$, причем если $H$ велико, то и $\nu$ велико. При больших $\nu$ имеем $\sigma(\nu) \leqslant\left(1+\varepsilon^{2}\right) \sigma(\nu-1)$ и, поскольку для $\nu-1$ неравенство $(17)$ уже неверно, получаем оценку

$$
H^{-\alpha_{s-1}} \leqslant \exp \left(-\frac{\tau_{2}+\delta^{-1}-\alpha_{s-1}\left(r_{1}+r_{2}+\varepsilon / 2\right)}{1+\varepsilon^{2}} \sigma(\nu)\right) .
$$

Рассмотрим соответствующую линейную форму $L_{\nu}(x)$ и определяемую ею гиперплоскость $M_{\nu}=\left\{x \mid L_{\nu}(x)=0\right\}$. Предположим, что доказаны соотношения

$$
\begin{gathered}
\rho\left(\theta, M_{\nu}\right) \leqslant \frac{1}{2}\left(H_{c}\left(M_{\nu}\right) H\right)^{-\alpha_{s-1}}, \\
\rho\left(\theta, M_{\nu}\right) \geqslant H^{-\alpha_{s}} .
\end{gathered}
$$

Тогда из соотношений $(20)$ и (3) следует, что $\rho\left(\theta, M_{\nu}\right)>\rho(\theta, N)$, т.е. заведомо $N \not \subset M_{\nu}$. Поэтому применимо следствие из предложения 3 и согласно (19)

$$
\begin{aligned}
\rho\left(\theta, M_{\nu} \cap N\right) H_{c}\left(M_{\nu} \cap N\right) & \leqslant H_{c}\left(M_{\nu}\right) H\left(\rho(\theta, N)+\rho\left(\theta, M_{\nu}\right)\right) \\
& <2 H \cdot H_{c}\left(M_{\nu}\right) \rho\left(\theta, M_{\nu}\right) \leqslant\left(H_{c}\left(M_{\nu}\right) H\right)^{1-\alpha_{s-1}} .
\end{aligned}
$$

$\mathrm{C}$ другой стороны, $\operatorname{dim}_{\mathscr{K}}\left(M_{\nu} \cap N\right)=s-1$, поэтому применимо предположение индукции. Определив $H^{\prime}$ из соотношения

$$
\left(H^{\prime}\right)^{\alpha_{s-1}}=H_{c}\left(M_{\nu} \cap N\right)\left(H_{c}\left(M_{\nu}\right) H\right)^{\alpha_{s-1}-1},
$$

мы видим, что $H_{c}\left(M_{\nu} \cap N\right) \leqslant H^{\prime}$, так как $H_{c}\left(M_{\nu} \cap N\right) \leqslant H_{c}\left(M_{\nu}\right) H$ (см. следствие из предложения 3). Поскольку $H^{\prime}$ при больших $H$ также велико, имеем

$$
\rho\left(\theta, M_{\nu} \cap N\right) \geqslant\left(H^{\prime}\right)^{-\alpha_{s-1}}=\left(H_{c}\left(M_{\nu}\right) H\right)^{1-\alpha_{s-1}} H_{c}\left(M_{\nu} \cap N\right)^{-1}
$$

что противоречит неравенству (21).

Итак, остается проверить неравенства (19) и (20).

Докажем (19). Применяя последовательно неравенства (11), (12) и оценки (1), имеем

$$
\begin{aligned}
& H_{c}\left(M_{\nu}\right)^{\alpha_{s-1}} \rho\left(\theta, M_{\nu}\right) \leqslant n^{d / 2} H_{c}\left(M_{\nu}\right)^{\alpha_{s-1}-\delta^{-1}}\left|L_{\nu}(\theta)\right| H\left(L_{\nu}\right)^{\left(r_{1}+r_{2}-1\right) / \delta} \\
& \quad \leqslant n^{d\left(1+\alpha_{s-1}-\delta^{-1}\right) / 2}\left|L_{\nu}(\theta)\right| H\left(L_{\nu}\right)^{\alpha_{s-1}\left(r_{1}+r_{2}\right)-\delta^{-1}} \\
& \quad \leqslant n^{d / 2\left(1+\alpha_{s-1}-\delta^{-1}\right)} \exp \left(-\left(\tau_{2}-\alpha_{s-1}\left(r_{1}+r_{2}\right)+\frac{1}{\delta}\right) \sigma(\nu)+\left(\tau_{2} \sigma(\nu)-\sigma_{2}(\nu)\right)\right) .
\end{aligned}
$$


Далее, $\sigma(\nu)$ при больших $H$ также велико, поэтому можно считать, что

$$
\tau_{2} \sigma(\nu)-\sigma_{2}(\nu) \leqslant \frac{\varepsilon}{4} \alpha_{s-1} \sigma(\nu)
$$

и оцениваемое выражение в силу (17) не превосходит

$$
n^{d\left(1+\alpha_{s-1}\right) / 2} e^{-\varepsilon \alpha_{s-1} \sigma(\nu) / 4} H^{-\alpha_{s-1}} \leqslant \frac{1}{2} H^{-\alpha_{s-1}} .
$$

Докажем (20). Применяя последовательно оценку (18), соотношения (2), неравенства $\alpha_{s} \geqslant 1$ и

$$
0<\varepsilon<\frac{1}{6\left(\tau_{1}+\delta^{-1}+1\right)}
$$

а также оценки (1), имеем

$$
\begin{aligned}
H^{-\alpha_{s}} & \leqslant \exp \left(-\frac{\tau_{2}+\delta^{-1}-\alpha_{s-1}\left(r_{1}+r_{2}+\varepsilon / 2\right)}{1+\varepsilon^{2}} \frac{\alpha_{s}}{\alpha_{s-1}} \sigma(\nu)\right) \\
& =\exp \left(-\frac{\tau_{1}+\delta^{-1}+\varepsilon \alpha_{s} / 2}{1+\varepsilon^{2}} \sigma(\nu)\right) \\
& <e^{-\left(\tau_{1}+\delta^{-1}+\varepsilon / 3\right) \sigma(\nu)} \leqslant\left|L_{\nu}(\theta)\right| H\left(L_{\nu}\right)^{-\delta^{-1}} e^{-\varepsilon \sigma(\nu) / 3}+\left(\sigma_{1}(\nu)-\tau_{1} \sigma(\nu)\right)
\end{aligned}
$$

Поскольку $\sigma(\nu)$ при больших $H$ велико и $\sigma_{1}(\nu)-\tau_{1} \sigma(\nu) \leqslant \varepsilon \sigma(\nu) / 6$, при больших $H$ оцениваемое выражение не превосходит

$$
\left|L_{\nu}(\theta)\right| H\left(L_{\nu}\right)^{-\delta^{-1}} e^{-\varepsilon \sigma(\nu) / 6} \leqslant n^{1 / 2} e^{-\varepsilon \sigma(\nu) / 6} \rho\left(\theta, M_{\nu}\right)<\rho\left(\theta, M_{\nu}\right)
$$

согласно неравенству (13).

Теорема полностью доказана.

Автор благодарит профессора Ю. В. Нестеренко за постановку задачи и ценные советы, а также профессора Д. Бертрана за полезные обсуждения.

\section{СПИСОК ЦИТИРОВАННОЙ ЛИТЕРАТУРЫ}

[1] Чудновский Г.В. Аналитические методы в диофантовых приближениях. Препринты № 74.8, № 74.9. Киев: ИМ АН УССР, 1974.

[2] Ressat E. Un critère d'indépendance algébrique // J. Reine Angew. Math. 1981. V. 329. P. 66-81.

[3] Waldschmidt M., Zhu Yao Chen. Une généralisation en plusieures variables d'un critère de transcendance de Gelfond // C. R. Acad. Sci. Paris. Sér. I. Math. 1983. V. 297. P. 229-232.

[4] Нестеренко Ю. В. Об одном достаточном признаке алгебраической независимости чисел // Вестн. МГУ. Сер. 1. Матем., мех. 1983. № 4. С. 63-68.

[5] Phillipon P. Critères pour l'indépendance algébrique de familles de nombres // C. R. Math. Rep. Acad. Sci. Canada. 1984. V. 6. P. 285-290.

[6] Нестеренко Ю. В. О мере линейной независимости чисел // Вестн. МГУ. Сер. 1. Матем., мex. 1985. № 1. С. 46-49.

[7] Гантмахер Р. Теория матриц. М.: Наука, 1986.

[8] Ленг С. Основы диофантовой геометрии. М.: Мир, 1986.

[9] Каган В.Ф. Основания теории определителей. М., 1921. 